\title{
Supporting Information:
}

\section{S1. FTIR}

We used FTIR to probe the binding of antibiotic at the surface of the gold nanoparticles. Gold nanoparticles were dried out of aqueous solution to form powder and mixed with $\mathrm{KBr}$. The spectrum was recorded on a Perkin Elmer Spectrum One FTIR Spectrometer operated in the diffuse reflectance mode at a resolution of $4 \mathrm{~cm}^{-1}$. To obtain good signal to noise ratio, 64 scans were taken in the range $450-4000 \mathrm{~cm}^{-1}$. For comparison, an FTIR spectrum of the cephalexin prepared in $\mathrm{KBr}$ pellet was also recorded. We have shown FTIR data (Figure below) recorded from Cephalexin (curve 1) and gold nanoparticles bound to Cephalexin (curve 2) recorded in $\mathrm{KBr}$ pellet. A number of bands can be clearly seen in curve 1 . The carbonyl group in the $\beta$-lactam ring occurs as a broad band centered at ca. $1780 \mathrm{~cm}^{-1}$, free carboxyl group at $1700 \mathrm{~cm}^{-1}$ and the carbonyl in amide linkage at $1650 \mathrm{~cm}^{-1}$ respectively.

However, we observed a remarkable change in the FTIR spectra after the formation of gold particles. The characteristic features of cephalexin are not evident which may be because of extremely low working concentration of the molecule, cephalaxin on the nanoparticle or the molecule may be broken as a result of the oxidation of the lactam ring. The data from this FTIR is not conclusive. 


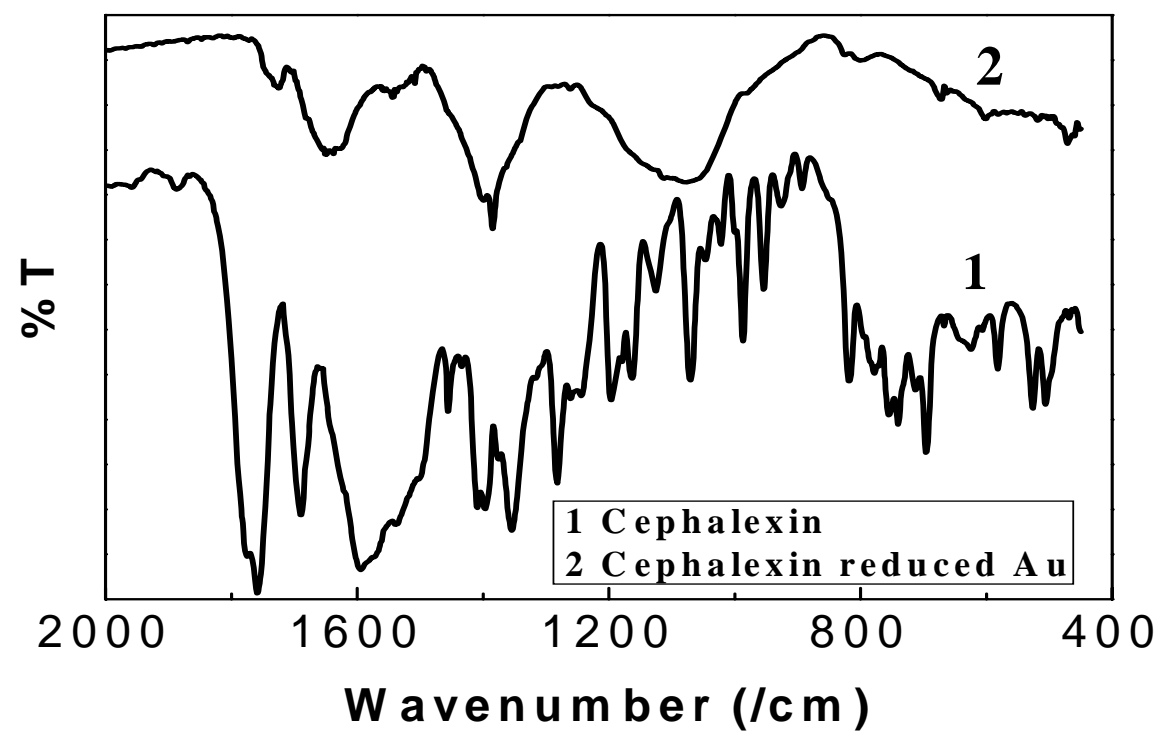

Figure S1: FTIR spectra of (1) cephalexin (2) gold nanoparticles bound with cephalexin. 


\section{S2. NMR spectrum of cephalexin}

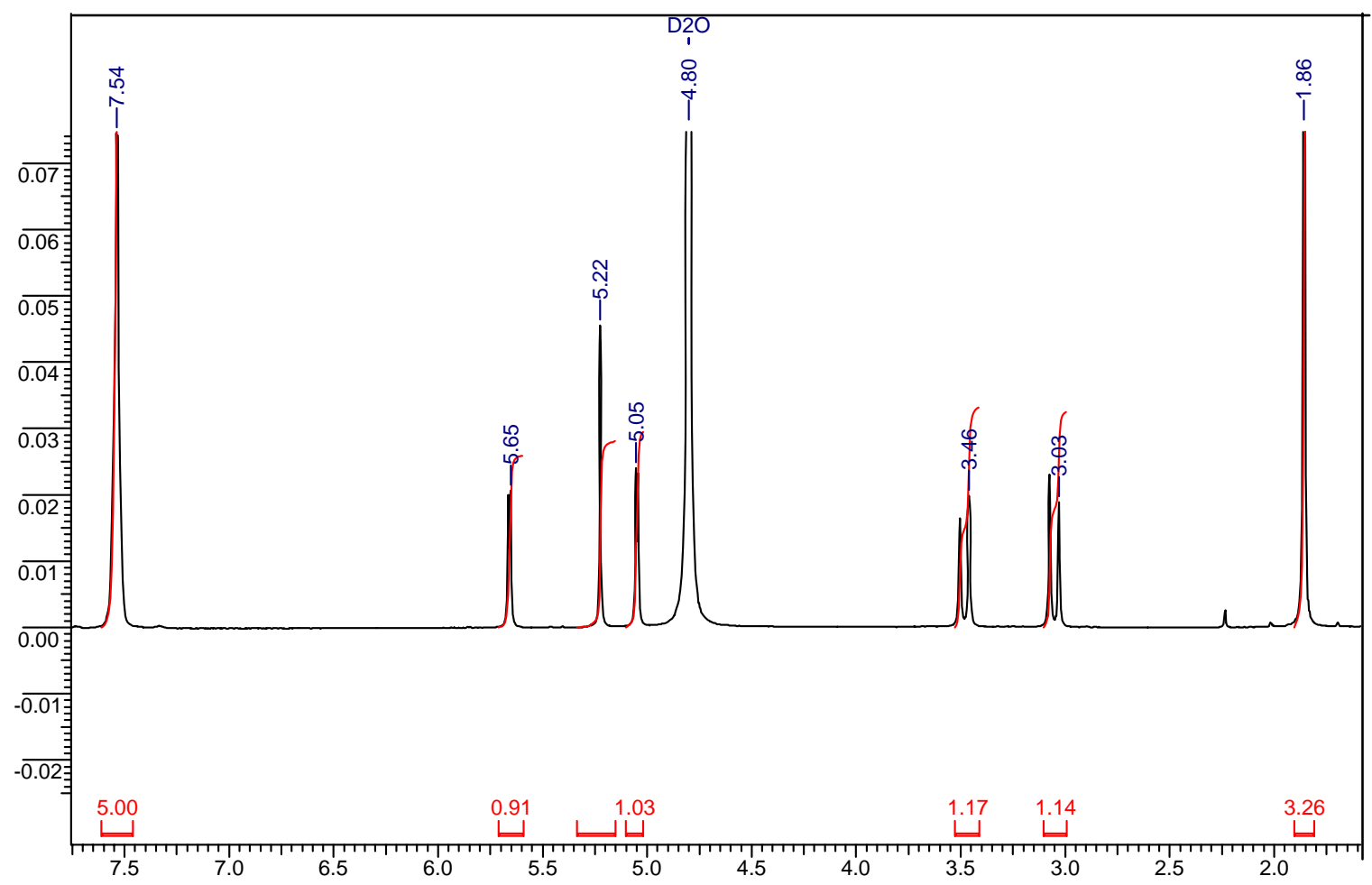

Figure S2: ${ }^{1} \mathrm{H}$ NMR spectrum of cephalexin.

${ }^{1} \mathrm{H}$ NMR (400 MHz, D $\left.2 \mathrm{O}\right): \delta 1.86(\mathrm{~s}, 3 \mathrm{H}), 3.03(\mathrm{~d}, 1 \mathrm{H}, \mathrm{J}=17.95 \mathrm{~Hz}), 3.46(\mathrm{~d}, 1 \mathrm{H}, \mathrm{J}=17.86 \mathrm{~Hz})$, $5.05(\mathrm{~d}, 1 \mathrm{H}, \mathrm{J}=4.29 \mathrm{~Hz}), 5.22(\mathrm{~s}, 1 \mathrm{H}), 5.65(\mathrm{~d}, 1 \mathrm{H}, \mathrm{J}=4.29 \mathrm{~Hz}), 7.54(\mathrm{~m}, 5 \mathrm{H})$

As evident from the above NMR spectrum, the cephalexin used in the study is pure and free from any other contamination. 


\section{S3. Electrophoresis of nanoparticles}

The nanoparticles were subjected to electrophoresis $(0.8 \%$ agarose gel) in $1 \mathrm{X}$ TAE buffer. The figure below shows the migration of the nanoparticles towards anode after 90mins under an electric field at $30 \mathrm{~V}$ (with respect to the bromophenol blue dye reference) showing that they are negatively charged.

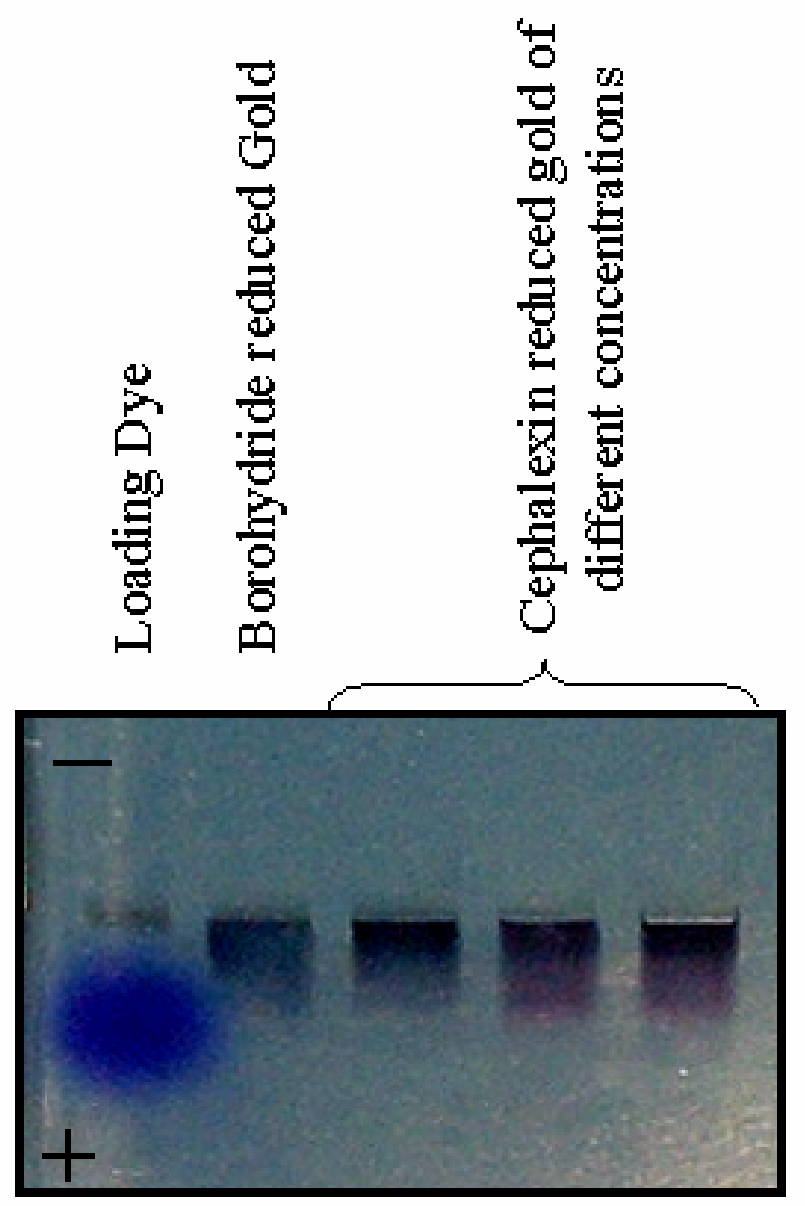

